J. Klin. Endokrinol. Stoffw. 2018 $11: 118-121$ https://doi.org/10.1007/s41969-018-0046-3 Online publiziert: 31. Oktober 2018 (c) Der/die Autor(en) 2018

CrossMark

\section{Sabina Smajis · Michael Krebs}

Klinik für Innere Medizin III, Abteilung für Endokrinologie und Stoffwechsel, Medizinische Universität Wien, Wien, Österreich

\title{
Postprandiale Hypoglykämie nach Magenbypass
}

Übergewicht ist ein weltweit zunehmendes Gesundheitsproblem. In westlichen Ländern sind 10-30\% der erwachsenen Personen übergewichtig [1]. Die progrediente Expansion der Adipositas in den letzten Jahrzehnten ist verantwortlich für erhöhte Prävalenz von Diabetes, kardiovaskulären Ereignissen [1], aber auch psychiatrischen Komorbiditäten in der Bevölkerung [2]. Neben einer Lebensstilmodifikation, die viel Disziplin erfordert und oft keine guten Langzeitergebnisse zeigt, hat sich die bariatrische Chirurgie, insbesondere der Roux-en-Y Gastric Bypass (RYGB) [3], als etablierte Therapieoption erwiesen. Bariatrische Operationen spielen somit eine zunehmend wichtigere Rolle in der Therapie der morbiden Adipositas. Die Gewichtsabnahme nach Magenbypassoperation wird einerseits durch die Restriktion des Nahrungsvolumens und Malabsorption, andererseits aber auch durch die Aktivierung der sättigungs- und appetitregulierenden intestinalen Peptide unterstützt [4]. Die Effekte der bariatrischen Operation auf die Gewichtsabnahme sowie auf die Verbesserung der adipositasassoziierten metabolischen Komplikationen sind klinisch bedeutend und inkludieren metabolische Effekte wie z. B. verbesserte Insulinsekretion oder sogar Diabetesremission [5] und positive Auswirkungen auf die Lebensqualität [6].

Allerdings ist die bariatrische Chirurgie, wie auch alle anderen invasiven Verfahren, mit einem gewissen sowohl peri- als auch postoperativen Risiko verbunden. Auch die Langzeitkomplikationen können in Einzelfällen schwerwiegend sein und reichen von erneuter Gewichtszunahme, Neuropathie, Hernien, Mängeln an wichtigen Mikronährstoffen bis zur noch nicht komplett erforschten postprandialen Hypoglykämie [4].

Die postprandiale Hypoglykämie sollte im Rahmen der klinischen Evaluation von einem frühen Dumpingsyndrom unterschieden werden. Der Verlauf der Glukosekonzentrationen nach einer kohlenhydratreichen Mahlzeit nach Magenbypass ist charakterisiert durch normale Nüchtern-Plasmaglukosespiegel, einen starken und schnellen postprandialen Anstieg von Glukose, Insulin und C-Peptid sowie einen daran anschließenden schnellen Abfall der Glukosekonzentration mit zum Teil deutlichen Hypoglykämien. Die Beschwerden entwickeln sich oft erst Monate bis einige Jahre postoperativ und treten meistens eine bis drei Stunden postprandial auf $[4,7]$. Im Gegensatz dazu entwickelt sich ein frühes Dumpingsyndrom unmittelbar postoperativ, tritt meistens innerhalb der ersten Stunde nach Nahrungsaufnahme auf und wird von einer Reihe autonomer hypoglykämieähnlicher Symptome begleitet [7].

Die postprandialen Hypoglykämien können in Einzelfällen schwer verlaufen und mit bedrohlichen Symptomen wie Bewusstseinsstörungen und Krampfanfällen einhergehen. In diesen Fällen ist mit einer relevanten Gefährdung unserer PatientInnen zu rechnen [4]. Aus der Behandlung von PatientInnen mit Diabetes mellitus ist bekannt, dass die sympathische Gegenregulation und damit die wichtigen Warnsymptome von Hypoglykämien bei häufigen Unterzuckerungen abnehmen können, was das Gefährdungspotenzial noch erhöht.

Im Gegensatz zur letztlich durch eine überschießende Insulinwirkung bedingten postprandialen Hypoglykämie stehen beim frühen Dumpingsyndrom andere Mechanismen im Vordergrund. Die durch den postoperativen Verlust der Reservoirfunktion schnelle Magenentleerung führt $\mathrm{zu}$ einer Distension des Dünndarms durch Übertritt von hyperosmolarem Speisebrei. Die nachfolgende Flüssigkeitsverschiebung aufgrund des osmotischen Gradienten kann zu Symptomen wie Hypotension mit Kollapsneigung und Sympathikusaktivierung führen [7].

\section{Diagnostik}

Die bisher verwendeten diagnostischen Tests geben keine klare Aussage über die echte Prävalenz der postprandialen Hypoglykämie nach bariatrischen Eingriffen. So wurde vergleichsweise eine Prävalenz der postprandialen Hypoglykämie bei $72 \%$ der bariatrisch operierten Patienten nach einem oralen Glukosetoleranztest (oGTT) und bei $29 \%$ der bariatrisch operierten Patienten nach einem flüssigen Mixed-MealTest berichtet. Krankenhausaufnahmen aufgrund von schweren postprandialen Hypoglykämien sind jedoch nur bei 0,2 \% der bariatrisch operierten PatientInnen nötig [8-10].

Ein oGTT mit Verabreichung von $75 \mathrm{~g}$ Glukoselösung in kurzer Zeit ist ein unphysiologischer Stimulus und kann eine Hypoglykämie bei Patienten mit der Anamnese eines chirurgischen Eingriffs im Bereich des oberen Gastrointestinaltrakts induzieren. Wichtig ist auch zu erwähnen, dass bei ca. $10 \%$ der gesunden Individuen Blutzuckerwerte von $<55 \mathrm{mg} / \mathrm{dl}$ ohne Hypoglykämie-Symptomatik nach einem oGTT auftreten können. Aus den genannten Gründen ist der oGTT als dia- 
gnostischer Test bei Verdacht auf postbariatrische Hypoglykämie nicht geeignet. Der in der Literatur beschriebene flüssige Mixed-Meal-Test könnte einen höheren diagnostischen Wert haben, da nicht nur Glukose, sondern auch Proteine und Lipide zugeführt werden. Allerdings ist auch eine flüssige Mahlzeit kein gutes Modell für die alltägliche Nahrungsaufnahme. In den bisher durchgeführten, sowohl flüssigen als auch festen Mahlzeitentests variiert die Kohlenhydratmenge zwischen 40 und 75 g pro Mahlzeit, und es gibt bisher keinen standardisierten und akzeptierten Mahlzeiten-Test, der diagnostisch, aber auch therapeutisch im klinischem Alltag zum Einsatz kommt [7, $8]$.

\section{Mechanismen}

Hypoglykämien nach bariatrischen Operationen sind multifaktoriell bedingt und noch nicht komplett erforscht. Eine wichtige Ursache ist sicher die inkretininduzierte inadäquate postprandiale Hyperinsulinämie [11].

Inkretine wie GLP-1 (Glucagon-like Peptide) und GIP (Gastric inhibitory Peptide) sind gastrointestinale Peptide, die zu einer stimulierten postprandialen Insulinsekretion aus den Beta-Zellen beitragen. Bis $\mathrm{zu} \mathrm{10-fach} \mathrm{höhere} \mathrm{post-}$ prandiale Konzentrationen von GIP und GLP-1 wurden bei bariatrisch operierten Patienten gemessen [4]. Stark erhöhte postprandiale Insulinspiegel, die verantwortlich für die postprandialen Hypoglykämien bei bariatrisch operierten Patienten sind, scheinen aus dieser stark erhöhten Inkretinausschüttung zu resultieren und werden nach intravenöser Glukosegabe nicht beobachtet [12]. Außerdem zeigte die Antagonisierung des GLP-1 durch eine kontinuierliche Infusion von Exenidin 9-39 einen höheren Effekt auf die Reduktion des postprandialen Insulinanstiegs bei RYGB-Operierten im Vergleich zu Nichtoperierten [13]. Neben der geänderten gastrointestinalen Anatomie könnten auch erhöhte zirkulierende Gallensäuren eine Rolle spielen. In seltenen Fällen könnte eine Inselzellhyperplasie (Nesidioblastose) auftreten [4]. Diese konnte in histologischen Pankreasuntersuchungen von pankreatek-

J. Klin. Endokrinol. Stoffw. 2018 ·11:118-121 https://doi.org/10.1007/s41969-018-0046-3 c) Der/die Autor(en) 2018

\section{S. Smajis · M. Krebs}

\section{Postprandiale Hypoglykämie nach Magenbypass}

\section{Zusammenfassung}

Bariatrische Operationen haben sich in der Behandlung der Adipositas als Therapieoption bewährt. Zu den postoperativen Risiken gehört neben dem Dumpingsyndrom auch die postprandiale Hypoglykämie. Sie entwickelt sich oft erst einige Monate bis Jahre nach der Operation und kann in Einzelfällen mit bedrohlichen Symptomen wie Bewusstseinsstörungen und Krampfanfällen einhergehen. Als eine wichtige Ursache gilt die inkretininduzierte inadäquate postprandiale Hyperinsulinämie. Auch erhöhte präoperative Konzentrationen von Insulin-like Growth Factor 1 (IGF-1) können mit einem erhöhten Risiko für die postprandiale Hypoglykämie verbunden sein.

\section{Postprandial hypoglycemia after gastric bypass surgery}

\section{Abstract}

Bariatric surgery has proved successful as a treatment option for obesity. Dumping syndrome and postprandial hypoglycemia are among the postoperative risks. The latter often develops several months to years after surgery and in individual cases it may be associated with life-threatening symptoms such as loss of consciousness and seizures. A significant cause is impaired incretininduced postprandial hyperinsulinemia. Raised preoperative concentrations of insulinlike growth factor 1 (IGF-1) may also be linked to an increased risk of postprandial hypoglycemia.

tomierten bariatrischen Patienten mit neuroglykopener Symptomatik nachgewiesen werden. Im Gegensatz dazu konnte in anderen histologischen Untersuchungen keine Beta-Zell-Hyperplasie, aber ein vergrößerter Zellkerndurchmesser der Beta-Zellen gezeigt werden. Dieses könnte ein Zeichen der Hyperfunktionalität sein und könnte erklären, warum es zu keiner kompletten Remission der Hypoglykämien nach partieller Pankreasresektion kommt [4].

Die Diskrepanz zwischen postprandialem Insulinanstieg und zeitlichem Auftreten von Hypoglykämien könn-
Grundlage der Therapie ist eine intensive diätologische Betreuung mit Identifikation der auslösenden Mahlzeiten. Schnell resorbierbare Kohlehydrate sollten generell vermieden werden. Medikamente wie Acarbose können den postprandialen Glukoseanstieg reduzieren und damit den Stimulus für die Insulinsekretion minimieren. Insgesamt ist die postprandiale Hypoglykämie noch nicht ausreichend erforscht; die Entwicklung standardisierter diagnostischer Tests ist dringend erforderlich.

Schlüsselwörter

Adipositas - Bariatrische Chirurgie .

Dumpingsyndrom · Hyperinsulinämie . Inkretine
The basis of treatment is intensive dietary supervision, with identification of the triggering foods. Fast-absorbing carbohydrates should generally be avoided. Medications such as acarbose can reduce the postprandial increase in glucose, thus minimizing the stimulus for insulin secretion. Overall, postprandial hypoglycemia has not yet been sufficiently studied; the development of standardized diagnostic tests is urgently required.

Keywords

Obesity · Bariatric surgery · Dumping syndrome $\cdot$ Hyperinsulinemia $\cdot$ Incretins 
gativ mit postoperativen Plasmaglukosewerten $2 \mathrm{~h}$ nach oraler Glukosegabe [11].

Postprandiale Hypoglykämie ist ein noch nicht ausreichend erforschtes Phänomen, und weitere pathophysiologische Studien sind erforderlich, um die therapeutischen Strategien zu verbessern. Auch die Entwicklung von standardisierten diagnostischen Tests ist dringend notwendig.

\section{Therapeutische Optionen}

Grundlage der Behandlung ist eine intensive diätologische Betreuung mit Identifikation der auslösenden Mahlzeiten z.B. mithilfe eines Diät- und Symptomtagebuchs. Generell werden das Meiden von rasch resorbierbaren Kohlenhydraten und die Aufteilung auf mehrere kleinere Mahlzeiten empfohlen. Durch die Verlangsamung der Kohlenhydratabsorption können Medikamente wie z.B. Acarbose den postprandialen Glukoseanstieg reduzieren und somit den Stimulus für die Insulinsekretion minimieren. Neben Acarbose können schwere postprandiale Hypoglykämien auch mit Octreotid oder Diazoxid behandelt werden [4]. Allerdings sind diese Therapien nicht bei allen Patienten ausreichend.

\section{Korrespondenzadresse}

\section{Dr. Sabina Smajis}

Klinik für Innere Medizin III, Abteilung für

Endokrinologie und Stoffwechsel, Medizinische Universität Wien

Währinger Gürtel 18-20, 1090 Wien, Österreich sabina.smajis@meduniwien.ac.at

Funding. Open access funding provided by Medical University of Vienna.

\section{Einhaltung ethischer Richtlinien}

Interessenkonflikt. S. Smajis und M. Krebs geben an, dass kein Interessenkonflikt besteht.

Dieser Beitrag beinhaltet keine von den Autoren durchgeführten Studien an Menschen oder Tieren.

Open Access. Dieser Artikel wird unter der Creative Commons Namensnennung 4.0 International Lizenz (http://creativecommons.org/licenses/by/4.0/deed. de) veröffentlicht, welche die Nutzung, Vervielfältigung, Bearbeitung, Verbreitung und Wiedergabe in jeglichem Medium und Format erlaubt, sofern
Sie den/die ursprünglichen Autor(en) und die Quelle ordnungsgemäßnennen, einen Linkzur Creative Commons Lizenz beifügen und angeben, ob Änderungen vorgenommen wurden.

\section{Literatur}

1. Anderwald $\mathrm{CH}$, Tura $A$, Promintzer-Schifferl $M$, Prager G, Stadler M, LudvikB et al (2012) Alterations in gastrointestinal, endocrine, and metabolic processes after bariatric Roux-en- $Y$ gastric bypass surgery. Diabetes Care 35(12):2580-2587

2. Simon GE, Von Korff M, Saunders K, Miglioretti DL, Crane PK, Van Belle G et al (2006) Association between obesity and psychiatric disorders in the US adult population. Arch Gen Psychiatry 63(7):824-830

3. Doble B, Wordsworth S, Rogers CA etal (2017) What Are the Real Procedural Costs of Bariatric Surgery? A Systematic Literature Review of Published Cost Analyses. Obes Surg 27(8):2179-2192. https://doi. org/10.1007/s11695-017-2749-8

4. Patti ME, Goldfine AB (2010) Hypoglycaemia following gastric bypass surgery-diabetes remission in the extreme? Diabetologia 53(11):2276-2279

5. Mingrone $G$ (2008) Role of the incretin system in the remission of type 2 diabetes following bariatric surgery. Nutr Metab Cardiovasc Dis 18(8):574-579

6. Versteegden DPA, Van Himbeeck MJJ, Nienhuijs SW (2018) Improvement in quality of life after bariatric surgery: sleeve versus bypass. Surg Obes RelatDis 14(2):170-174

7. Emous M, Ubels FL, van Beek AP (2015) Diagnostic tools for post-gastric bypass hypoglycaemia. Obes Rev 16(10):843-856

8. Marsk $R$, Jonas $E$, Rasmussen $F$, Näslund $E$ (2010) Nationwide cohort study of post-gastric bypass hypoglycaemia including 5,040 patients undergoing surgery for obesity in 1986-2006 in Sweden. Diabetologia 53(11):2307-2311

9. Roslin M, Damani T, Oren J, Andrews R, Yatco E, Shah $P$ (2011) Abnormal glucose tolerance testing following gastric bypass demonstrates reactive hypoglycemia. Surg Endosc 25(6):1926-1932

10. Kefurt R, Langer FB, Schindler K, ShakeriLeidenmuhler S, Ludvik B, Prager G (2015) Hypoglycemia after Roux-En-Y gastric bypass: detection rates of continuous glucose monitoring (CGM) versus mixed meal test. Surg Obes Relat Dis 11(3):564-569

11. Itariu BK, Zeyda M, Prager G, Stulnig TM (2014) Insulin-like growth factor 1 predicts post-load hypoglycemia following bariatric surgery: a prospective cohort study. PLoS ONE 9(4):e94613

12. Toft-Nielsen M, Madsbad S, Holst JJ (1998) Exaggerated secretion of glucagon-like peptide1 (GLP-1) could cause reactive hypoglycaemia. Diabetologia 41(10):1180-1186

13. Salehi M, Vella A, McLaughlin T, Patti ME (2018) Hypoglycemia after gastric bypass surgery: current concepts and controversies. J Clin Endocrinol Metab 103(8):2815-2826

\section{ICCBH 2019}

$9^{\text {th }}$ International Conference on Children's Bone Health

22-25 June 2019, Salzburg, Austria

In Association with the

European Calcified Tissue Society (ECTS)

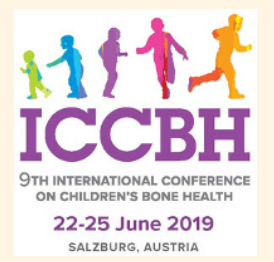

\section{Scientific programme}

Key topics: Bone development - Bone fragility and metabolism $\cdot$ Bone diagnostics - Bone health in chronic and rare diseases - Epidemiology • Novel drug targets and medical therapies $•$ Nutrition and rickets • Orthopaedics - Physical activity and biomechanics

Learning objectives: Inherited and acquired bone disorders - Effects of chronic illness on muscle and bone - Bone therapeutics $\cdot$ Bone diagnostics $\cdot$ Bone signalling and metabolism

Deadline for abstracts:

11 February 2019

Deadline for early registration: 22 March 2019

www.iccbh.org

SAVE THE DATE - ESPE 2019

$5^{\text {th }}$ Annual European Society for Paediatric Endocrinology Meeting 19-21 September 2019, Vienna, Austria www.eurospe.org

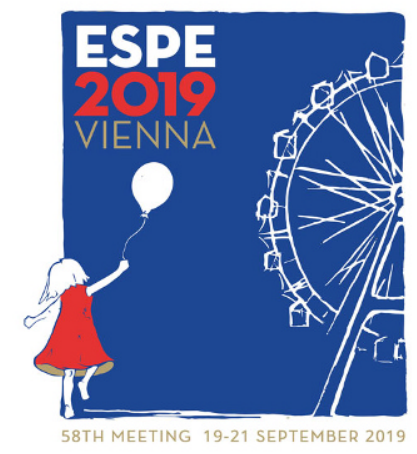


Hier steht eine Anzeige.

\section{曾 Springer}

